\title{
Aspectos fundamentales del método de Edith Stein ${ }^{1}$
}

\author{
Mariano Crespo \\ FACULTAD DE FILOSOFÍA \\ PONTIFICIA UNIVERSIDAD CATÓLICA DE CHILE
}

\section{$I$}

El 3 de Mayo de 1917 Edmund Husserl imparte su primera lección en la Universidad de Friburgo. En dicha lección, titulada La fenomenología pura, su ámbito de investigación y su método ${ }^{2}$, el filósofo germano plantea la necesidad de una profunda renovación de la filosofía europea de su tiempo. Frente a las Rennaissance-Philosophien (neokantismo, neohegelianismo, etc.), que intentan reformulaciones de sistemas filosóficos ya construidos y frente a las distintas formas de fenomenismo empirista que niegan la objetividad del conocimiento, la fenomenología surge como una filosofía completamente originaria, una nueva ciencia fundamental, una ciencia estricta y de una extensión infinita. No se trata aquí de un conjunto de proposiciones y verdades filosóficas en las que todo el que se dice «fenomenólogo» ha de creer a pies juntillas, sino, más bien, de un modo de filosofar que difiere de la forma habitual de tratar con las cosas y de la manera en que trabajan con ellas las ciencias fácticas. El punto de partida de este método son, por consiguiente, los datos de la experiencia y no teorías acerca de éstos. Se trata, pues, como afirma Max Scheler, de «un enfoque peculiar de la con-

1 Conferencia impartida el 7 de Mayo de 2010 en el seno del Simposio «Edith Stein. Hacia la pregunta por la mujer» organizado por la Facultad de Teología de la Pontificia Universidad Católica de Chile.

2 E. Hussert, «Die reine Phänomenologie, ihr Forschungsgebiet und ihre Methode. Freiburger Antrittsrede» en: E. Husserl, Aufsätz̨e und Vorträge (1911-1921). Mit ergänzenden Texten (Husserliana XXV, herausgegeben von T. Nenon und H.R. Sepp, Martinus Nijhoff, Dordrecht 1997, 68-81). 
templación espiritual a través del cual se obtiene una visión o una vivencia que quedarían ocultas sin este enfoque» ${ }^{3}$.

Pues bien, fue justamente esta forma de entender la filosofía, plasmada por primera vez en las Investigaciones Lógicas, la que atrajo a una serie de pensadores alemanes, gran parte de ellos discípulos del psicólogo muniqués Theodor Lipps, a desplazarse desde la capital de Baviera a Gotinga, ciudad ésta en la que Husserl era profesor desde el semestre de invierno de 1901-1902. A este círculo de Múnich pertenecían, entre otros, autores como Adolf Reinach, Johannes Daubert, Alexander Pfänder, Dietrich von Hildebrand, Moritz Geiger, Theodor Conrad y Hedwig Conrad-Martius. Los primeros de estos filósofos que llegaron a Gotinga fueron Reinach y Daubert (semestre de verano de 1905). Posteriormente se incorporaron Conrad (1907) y Hildebrand (1909). Más tarde, en 1913, una joven estudiante, no procedente del círculo de Lipps en Múnich, se convierte en un miembro importante de este grupo. Me refiero a Edith Stein.

En torno a 1907 estos estudiantes forman la Sociedad filosófica de Gotinga, presidida por Theodor Conrad, la cual solía reunirse una vez a la semana, fuera de las salas de clases, para discutir y leer artículos ${ }^{4}$. De hecho, Edith Stein cuenta que las reuniones tenían lugar los viernes por la tarde y que se prolongaban hasta las 2 o las 3 de la madrugada. Esto originaba que los estudiantes que asistían a las mismas acudieran al seminario que Husserl impartía los sábados por la mañana poco despiertos. Ante la falta de actividad mental de algunos de sus mejores estudiantes, Husserl les prohibió que se reunieran los viernes por la tarde ${ }^{5}$.

Como es de sobra conocido, Edith Stein siguió a Husserl a Friburgo donde se doctoró con una tesis sobre el problema de la empatía. Dado que nuestra autora permaneció como asistente de su maestro hasta el

3 M. SCHELER, «Phänomenologie und Erkenntnistheorie» en M. SCHELER, Gesammelte Werke (Bd. I. Schriften aus dem Nachlass. Francke Verlag, Bern 1957, 377 («Fenomenología y gnoseología» en M. SCHELER, La esencia de la filosofía y la condición moral del conocer filosófico (Ed. Nova, Buenos Aires 1980, 62).

4 H. Spiegelberg, The Phenomenological Movement. A Historical Introduction. Third revised and enlarged edition. With the collaboration of Karl Schuhmann, (Martinus Nijhoff, The Hague 1982, 166ss).

5 El texto de Edith Stein en el que se relata esta anécdota es citado por K. SCHUHmann, Husserl-Chronik. Denk- und Lebensweg Edmund Husserls (Martinus Nijhoff, Den Haag 1977). 
otoño de 1918, es muy probable que se contara entre los oyentes de esa lección inaugural de Husserl a la que me acabo de referir.

En este trabajo quisiera abordar - de un modo un tanto diverso a como habitualmente se suele hacer- tres aspectos, a mi juicio, fundamentales del método filosófico de Edith Stein. En primer lugar, me referiré al punto de partida del filosofar de nuestra autora. En segundo lugar, aludiré -con algo más de detenimiento- al aspecto que constituye uno de los aportes fundamentales del método fenomenológico y que, a mi modo de ver, es claramente reconocible en nuestra autora, a saber, el haber puesto de manifiesto la imposibilidad de hacer filosofía primera sin tomar en cuenta la vida consciente ante la que todas las cosas se abren. En tercer lugar, quisiera referirme a un aspecto de la antropología de Stein que considero especialmente relevante. Al final de mis análisis de cada uno de estos tres aspectos metodológicos quisiera mostrar en qué sentido pueden ser de relevancia para emprender el camino hacia la pregunta por la mujer.

Antes de abordar los tres aspectos fundamentales del método de Edith Stein que acabo de mencionar quisiera hacer una observación previa. Es común presentar el pensamiento de nuestra autora -tanto en sus aspectos metodológicos como de contenido- como dominado por una doble cesura: por un lado, por el alejamiento de la orientación idealista trascendental de Husserl y, por otro lado, por el hecho de su conversión al catolicismo. Ciertamente, Edith Stein, al igual que gran parte de los primeros discípulos de Husserl en Gotinga, perciben en su maestro un alejamiento del objetivismo de la primera edición de Investigaciones lógicas. Asimismo, la conversión lleva de la mano, entre otras muchas cosas, un adentramiento en una tradición filosófica diferente a aquella en la que Stein se había formado. Me refiero al tomismo. Piénsese, por ejemplo, en la traducción que realiza de las Quaestiones disputatae de veritate de Tomás de Aquino o en el conocimiento que del pensamiento del Doctor Angélico demuestra en Ser finito, ser eterno o en el artículo que, con motivo del 70 aniversario de Husserl, escribió y en el que emprende una comparación entre el pensamiento de éste y el del Aquinate. En virtud de estas dos «cesuras» las presentaciones de los aspectos metodológicos de nuestra autora suelen siempre centrarse en un antes y un después de cada una de ellas. De este modo, se insiste en los aspectos en los que el realismo de Stein diferiría del idealismo trascendental de Husserl y en la incompatibilidad entre este último y la filosofía de Tomás de Aquino. 
Ciertamente, existen diferencias importantes. Sin embargo, en este trabajo quisiera esforzarme en ofrecer una visión más integradora. Dicho en términos positivos, quisiera centrarme en los aspectos que, a mi juicio, hacen del modo de hacer filosofía de Edith Stein el método auténticamente filosófico.

\section{II}

En primer lugar, el punto de partida del filosofar en Edith Stein son las cosas mismas, los problemas y no las filosofías. Ciertamente, éstas ayudan a la identificación de los problemas que se trata de esclarecer, pero no libran de la necesidad de mirar con los propios ojos aquello que se intenta dilucidar. En los escritos de Stein resuenan las palabras de Aristóteles en De anima:

«Baste con lo anterior como noticia sobre las opiniones de nuestros predecesores sobre el alma. Dejémoslas a un lado y consideremos que estamos de nuevo en el punto de partida y tratemos de dar una respuesta precisa a la pregunta: ¿qué es el alma?»».

A este punto se refiere Husserl en su artículo programático La filosofía, ciencia rigurosa, publicado en $1910^{7}$. En ese trabajo el fundador del método fenomenológico insiste en que la filosofía, en cuanto ciencia de lo radical, tiene que ser ella misma en su procedimiento, radical. Esta radicalidad se traduce en no aceptar como verdadera ninguna tesis que no hayamos personalmente comprobado en la medida de nuestras fuerzas recurriendo al tipo de experiencia en el que auténticamente se tenga acceso a las cosas de las que se habla. Esta remisión a la experiencia explica que el punto de partida del método fenomenológico sean precisamente los fenómenos y su objetivo la descripción fiel de los mismos ${ }^{8}$. Ahora bien, como señala la propia Stein ${ }^{9}$, al fenomenólogo no le interesan los fenómenos en el senti-

6 Aristóteles, De anima, II.

7 «Philosophie als strenge Wissenschaft» en E. Husserl, Aufsätze und Vorträge (19111921). Mit ergänzenden Texten. (Herausgegeben von T. Nenon und H.R. Sepp, Husserliana XXV, Martinus Nijhoff, Dordrecht 1997, 61 (Filosofía, ciencia rigurosa. Trad. de Miguel García-Baró. Ediciones Encuentro, Madrid 2009, 85).

8 Cf. E. SteIn, Einführung in die Philosophie en E. SteIn, Werke. Bd. XIII, Herder, Freiburg 1991) 33 (Introducción a la filosofía, en Obras Completas, II, Escritos filosóficos (Etapa fenomenológica: 1915-1920, Ediciones El Carmen, Editorial de espiritualidad, Editorial Monte Carmelo, Burgos 2005, 684).

9 «Was ist Phänomenologie?» en Wissenschaft/Volksbindung, Neuen Pfälzischen Landeszeitung, n. 5, 15.05.1924, reimpr. Theologie und Philosophie, 66, 1991,. 570-573 («Che 
do habitual de las meras apariencias ni tampoco las vivencias individuales y singulares que se dan en cada caso. Le interesan estas últimas tan sólo en la medida en que en ellas es apresable su estructura esencial, siguiendo la terminología husserliana, «en pureza eidética ${ }^{10}$. En palabras de Stein:

«[...] no se trata de aprehenderlos [los fenómenos] sólo como fenómenos singulares y explicitar todo lo implícito en ellos, [...] sino de penetrar en su esencia. La fenomenología de la percepción no se conforma con describir la percepción singular, sino que quiere indagar lo que es "percepción en general", según su esencia, y obtiene este conocimiento del caso singular en abstracción ideante» ${ }^{11}$.

Lo que era considerado como un individuo pasa ahora a ser un ejemplar de una esencia. De este modo, como señala Husserl, el ente existente se transforma en un ente esencial, lo individualmente único en una generalidad. Nuestro interés no se concentra ahora en la vivencia individual, sino en la vivencia pura, es decir, en la esencia-de-la-vivencia captable en actitud eidética sobre la base de las aprehensiones empíricas. Por consiguiente, en la intuición eidética no se captan hechos singulares y existentes, sino esencias.

Este estilo radical de filosofar que intenta llegar a la verdad de las cosas, a describir los fenómenos está en las antípodas de las grandes síntesis y de las visiones panorámicas. Edith Stein juzgaba preferible comprender un solo punto con entera claridad que perderse en las brumas de una exposición generalista. Sus trabajos están animados por la búsqueda de la precisión, por el intento de «comprender con exactitud y formular con

cos'é la fenomenologia» en E. STEIN, La ricerca della verita. Dalla fenomenologia alla filosofia cristiana. A cura di A. Alles Bello, Citta Nuova, Roma 1993, 56).

10 «Wir folgen unserem allgemeinen Prinzip, dass jedes individuelle Vorkommnis sein Wesen hat, das in edetischer Reinheit fassbar ist und in dieser Reinheit zu einem Felde möglicher eidetischer Forschung gehören muss» (E. HusserL, Ideen zu einer reinen Phänomenologie, Erstes Buch, Allgemeine Einführung in die reine Phänomenologie, Hrsg. V. W. Biemel, Husserliana III/1, Martinus Nijhoff, The Hague 1950, 』 34, 60; «Es wird dann evident, dass jedes Erlebnis des Tromes, das der reflektive Blick zu treffen vremag, ein eigenes, intuitiv zu erfassendes Wesen hat, einen ,Inhalt', der sich in seiner Eigenheit für sich betrachten lässt» (E. HussERL, Ideen zu einer reinen Phänomenologie, Erstes Buch., S 34, 61).

11 E. STEIN, Zum Problem der Einfüblung (Reprint der Originalausgabe von 1917, Verlagsgesellschaft Gerhard Kaffke, München 1980, 2), (Sobre el problema de la empatía, en Obras Completas. Escritos filosóficos (Etapa fenomenológica: 1915-1920, Editorial de Espiritualidad, Editorial Monte Carmelo, Ediciones El Carmen, Burgos 2002, 80. 
claridad cada distinción, cada problema» ${ }^{12}$, por evitar cualquier tesis que no se nos imponga por los datos, por la abstención de presupuestos tácitos que ni son evidentes ni están probados, por el esfuerzo por un análisis sistemático en lugar de por la construcción de un sistema y por la renuncia a la sistematización precipitada ${ }^{13}$.

Es justamente este modo en que Husserl trataba los problemas filosóficos, ese afán de comprender y estudiar desde dentro en lugar de criticar desde arriba ${ }^{14}$, el que ganó a Edith Stein para la filosofía al igual que al mismo Husserl le sucedió con su maestro Franz Brentano. Es el mismo afán de radicalidad que, años más tarde, encontraría en los escritos de Tomás de Aquino. Tanto el filósofo germano como el Doctor Angélico consideraban a la filosofía como una ciencia rigurosa -la más rigurosa de todas- que trata de obtener una imagen del mundo lo más universal y fundamentada posible. Este afán compartido por el Aquinate y por Husserl (y en definitiva por todo auténtico filósofo) es formulado por nuestra autora del siguiente modo:

«Por lo tanto, en un punto se observa una completa concordancia: el de cultivar la filosofía como ciencia rigurosa, tal como la denominó Husserl. [...] No se trata de una analogía con alguna otra ciencia. Significa tan sólo que la filosofía no es cosa del sentimiento ni de la fantasía, no lo es de una exaltación de altos vuelos ni de una opinión personal; no es, como quien dice, cuestión de gusto, sino que es cosa de la razón que investiga seria y sobriamente. Tanto en Husserl como en Tomás de Aquino domina la convicción de que un logos gobierna todo cuanto es, de que es posible para nuestro conocimiento descubrir paso a paso algo de ese logos y de que siguiendo la máxima de la más estricta honradez intelectual, este conocimiento progresará cada vez más» $»^{15}$.

12 Es precisamente esta la actitud que -con justicia- ve L. Rodríguez Duplá en uno de los pensadores españoles contemporáneos más destacados, a saber, Juan Miguel Palacios. Cf. L. Rodríguez Duplá, «Una vocación universitaria» en I. García de LeÁNIZ (ed.), De nobis ipsis silemus. Homenaje a Juan Miguel Palacios (Ediciones Encuentro, Madrid 2010, 24).

13 Cf. D. v. Hildebrand, Ethik, Prolegomena (Gesammelte Werke, Bd. II, 2. Auflage, Verlag W. Kohlhammer, Stuttgart, 1973, 7-26); Ética, Prolegómenos. (Trad. de J.J. García Norro, Ediciones Encuentro, Madrid 1983, 13-30).

14 Cf. «Die reine Phänomenologie, ihr Forschungsgebiet und ihre Methode. Freiburger Antrittsrede», 81.

15 E. STEIN, «Was ist Philosophie? Ein Gespräch zwischen Edmund Husserl und Thomas von Aquin» en E. SteIn, Werke. Eds. L. Gelber y M. Linssen O.C.D., Vol. XV. 
Ciertamente, en este punto existen, al menos, dos diferencias importantes, entre el método fenomenológico en el que Edith Stein se formó y el método de la filosofía tomista al que se acercó sistemáticamente después de su conversión. Por un lado, mientras que la fenomenología trata de establecerse como ciencia de esencias para una conciencia, para Santo Tomás a las investigaciones de esencias deben añadirse los hechos de la experiencia natural y los que aporta la fe. Santo Tomás no admitiría -como sí lo admitiría Husserl- que el camino de la razón natural es el único camino del conocimiento. Frente a la concepción de que la tarea de la razón es infinita, el Aquinate sostiene que la verdad plena existe y que hay un conocimiento que puede abarcarla totalmente: es el conocimiento divino $^{16}$. Sin embargo, durante la existencia terrena se le comunica por la Revelación al espíritu algo de lo que verá cara a cara.

La relevancia de este aspecto metodológico fundamental en la pregunta por la mujer es, a mi juicio, doble. Por un lado, determina que esta pregunta ha de ser radical en el modo en el que aquí hemos utilizado este término. Será, ciertamente, de mucha utilidad recopilar los resultados de las principales teorías acerca de la condición femenina, pero, tarde o temprano, habrá que hacerse la pregunta -semejante a la que Aristóteles se hacia en el pasaje del De anima que hemos citado- ¿qué es la mujer? Por otro lado, la investigación a esta pregunta, si quiere ser auténticamente filosófica, ha de ser eidética. Parafraseando a Stein, no se trataría de aprehender los fenómenos singulares -si se me permite hablar así- femeninos, como fenómenos singulares y explicitar todo lo implícito en ellos, sino de penetrar en su esencia. La fenomenología de lo femenino -si cabe hablar así- no se conformaría con describir fenómenos singulares, sino que quiere indagar en lo que es 'lo femenino en general', según su esencia, obteniendo este conocimiento del caso singular en abstracción ideante. Se trataría, pues, de elaborar una teoría eidética de la mujer. En estrecha relación con este segundo aspecto, la investigación filosófica acerca de la mujer debería, a mi modesto entender, evaluar cuál es su relación con las ciencias empíricas. En un texto de clara inspiración husserliana como es su Introducción a la filosofía Edith Stein no parece especialmente optimista al respecto. Allí se señala que el método filosófico no debe asentarse sobre los resultados de cualquier ciencia positiva. «El terreno de la investigación

Erkenntnis und Glaube, Herder, Freiburg 1993, 19-48; ¿Qué es filosofía? Trad. de A. Valero Martín. Opuscula philosophica, Ediciones Encuentro, Madrid 2001, 14)

16 E. STEIN, op.cit, p. 153. 
de la filosofía debe ser un campo de absoluta certeza, de conocimiento irrevocable» ${ }^{17}$. Si esto es así, prosigue nuestra autora, «entonces no sólo hemos de descartar los resultados de las ciencias particulares, sino que además hemos de "poner entre paréntesis" todo lo que sabemos por experiencia» ${ }^{18}$. ¿No habría, pues, relación alguna entre el modo en el que las ciencias empíricas, por ejemplo, la psicología y la filosofía, en cuanto teoría eidética, se hacen la pregunta por la mujer? No son fáciles estas preguntas. Responder a ellas en detalle desborda los límites de esta conferencia y, sobre todo, de este conferenciante. Déjenme, sin embargo, aventurar un par de pensamientos.

A pesar de la clara diferencia que existiría entre una fenomenología de la mujer en cuanto teoría eidética y la psicología, cabe preguntarse si los resultados de aquella tienen algún tipo de repercusión en las investigaciones psicológicas. Con respecto a esto, pienso que puede decirse lo siguiente. Hemos visto que la ciencia eidética investiga la esencia mientras que la empírica investiga la existencia. El análisis de esencias es eo ipso análisis genérico, análisis de objetos universales. En este orden de cosas, Husserl sostiene que la ciencia eidética precede a la ciencia empírica ${ }^{19}$. En el caso concreto de las relaciones entre psicología y fenomenología, esto significa que la psicología supone lo que la fenomenología estudia. Por tanto, aquella no puede prescindir de lo que esta última descubre. Como afirma el fundador del método fenomenológico «las verdades eidéticas valen, y valen con universalidad absoluta, así para todo lo posible como para todo lo que en la experiencia actual se acredita como real» $»^{20}$. Por consiguiente, las verdades eidéticas no pueden ser transgredidas, confirmadas o refutadas mediante la experiencia ${ }^{21}$. Si un análisis psicológico parece «contradecir» el análisis fenomenológico, ello se debe a deficiencias en este último. La razón última de esto reside en que estas verdades eidéticas están referidas

17 «Das Forschungsgebiet der Philosophie soll ein Feld absoluter Gewissheit, unwiderruflicher Erkenntnis sein» (E. STEIN, Einführung in die Philosopbie, 31; Introducción a la filosofía, 681).

18 Ibid.

19 E. HusserL, Ideen zur einer reinen Phänomenologie und phänomenologischen Philosophie. Zweites Buch: Phänomenologische Untersuchungen zur Konstitution. (Hrsg. von Marly Biemel, Martinus Nijhoff, The Hague, 1952. \55).

20 Ibid.

21 E. HusserL, Ideen zur einer reinen Phänomenologie und phänomenologischen Philosophie. Drittes Buch: Die Phänomenologie und die Fundamente der Wissenschaften. (Hrsg. von Marly Biemel, Martinus Nijhoff, The Hague, 1971, \60) 
a las vivencias en sí mismas, por consiguiente, tanto a las actuales como a las posibles. Por otro lado, y estrechamente relacionado con esto, podemos decir que las verdades descubiertas en el análisis eidético enriquecen el conocimiento psicológico.

\section{III}

El segundo aspecto fundamental del método de Edith Stein al que quisiera referirme tiene que ver con una de las aportaciones, a mi juicio, más decisivas de la fenomenología. Me refiero al haber puesto de manifiesto la imposibilidad de hacer filosofía primera sin tomar en cuenta la vida consciente ante la que todas las cosas se abren. En este orden de cosas, Walter Biemel, en su conocido artículo sobre las tres fases de la fenomenología de Husserl, ha puesto de relieve que el hilo conductor del pensamiento todo de este autor no es sino la idea de que para iluminar la esencia de una cosa es necesario remontarse al origen de su significación en la conciencia y a la descripción de este origen ${ }^{22}$. De este modo, surge ante nosotros un gran ámbito de investigación, a saber, la región de la conciencia, entendida ésta, en palabras de Stein, como un «terreno, hasta entonces inexplorado, de investigación científica esencial» y como el «necesario punto de partida de toda investigación filosófica, el terreno absoluto que buscamos» $»^{23}$. Recuérdese cómo Husserl introduce la reducción fenomenológica a fin de hacer posible el volver la mirada hacia esta región de la conciencia pura o subjetividad ${ }^{24}$. De este modo, lograremos aislar las estructuras fundamentales de la subjetividad y evitar el error de la metabasis eis allo genos consistente en identificar la conciencia pura con la conciencia fáctica y, por ende, la fenomenología con la psicología ${ }^{25}$. La reducción no es concebida por Husserl como una negación o una conjetura o una duda, sino como una «purificación radical del campo fenomenológico de la conciencia de todas las intromisiones (Einbrüchen) de las realidades objetivas» ${ }^{26}$.

22 W. Biemel, «Las fases decisivas en el desarrollo de la filosofía de Husserl» (Husserl. Tercer Coloquio filosófico de Royaumont. Versión castellana supervisada por Guillermo Maci, Paidós, Buenos Aires 1968, 35-57).

23 E. STEIN, Einfübrung in die Philosophie, 32 (Introducción a la filosofía, 682).

24 Cf. E. Husserl, Ideen zu einer reinen Phänomenologie, Erstes Buch, \56.

25 E. Husserl, Ideen qu einer reinen Phänomenologie, Erstes Buch, \61.

26 E. Husserl, «Die reine Phänomenologie, ihr Forschungsgebiet und ihre Methode. Freiburger Antrittsrede», 75 
Radicalizando su consideración de la naturaleza de la conciencia, el fundador del método fenomenológico sostiene que si se produjera la aniquilación del mundo, (Weltvernichtung), la conciencia sobreviviría como ser absoluto, puro. Esto conlleva, por tanto, que la existencia de los objetos es contingente mientras que la de la conciencia es absoluta. En palabras del mismo Husserl:

«[...] ningún ser real en sentido estricto, ningún ser que se exhiba y compruebe mediante apariencias, es para el ser de la conciencia misma (en el más amplio sentido de corriente de vivencias) necesario. El ser inmanente es, pues, ya sin duda, ser absoluto en el sentido de por principio nulla ' $r$ ' indiget ad existendumo ${ }^{27}$.

De este modo, la conciencia pura queda caracterizada como un «orden del ser encerrado en sí», «un orden de ser absoluto» ${ }^{28}$, mientras que la realidad o el mundo tendrían un mero ser intencional ${ }^{29}$, un ser para una conciencia, abriéndose pues un verdadero abismo entre la conciencia y la realidad ${ }^{30}$.

A diferencia de lo que pudiera parecer a primera vista, no es aquí donde reside, en sentido propio, el inicio del alejamiento de nuestra autora -y de sus colegas de la Sociedad Filosófica de Gotinga- de su maestro al considerar que este último había iniciado la senda del idealismo. En un texto en el que Edith Stein se ocupa directamente de la argumentación husserliana que acabo de reproducir, ésta reconoce que la afirmación de un ser que no fuera experimentable no tendría sentido ${ }^{31}$. Podría suceder que lo que, en el fondo, significa la afirmación husserliana no es:

27 «Also kein reales Sein, kein solches, das sich bewusstseinsmässig durch Erscheinungen darstellt und ausweist, ist für das Sein des Bewusstseins selbst (im weitesten Sinne des Erlebnisstromes) notwendig. Das immanente Sein ist also zweifellos in dem Sinne absolutes Sein, das es prinzipiell nulla, re' indiget ad existendum». E. HusserL, Ideen zu einer reinen Phänomenologie, Erstes Buch, Hua III/1, \49, 60. La traducción es de J. GAOs (Ideas relativas a una fenomenología pura y una filosofía fenomenológica, FCE, Madrid 1985, 113).

28 E. Husserl,., Ideen zu einer reinen Phänomenologie, Erstes Buch, Hua III/1, \ 49, 93. Ibid.

30 E. HusserL, Ideen zu einer reinen Phänomenologie, Erstes Buch, \50, 102.

31 «Dass die Behauptung eines prinzipiell unerfassbaren Seins keinen Sinn hätte, wird man zugestehen müssen» (Einführung in die Philosophie, p. 94) La traducción al castellano oculta el auténtico sentido: «Habrá que admitir que la afirmación de un ser que, por principio, fuera imposible de captar, no tendría sentido» (Introducción a la filosofía, p. 747). 
«sino que la realidad no puede mostrarse de otra manera que no sea por medio de la experiencia, y que cualquier enunciado sobre la realidad no puede adquirir su legitimidad sino por medio de la experiencia» ${ }^{32}$.

Es justamente a este carácter mostrenco de la realidad a la conciencia a la que se refiere la problemática fenomenológica de la constitución. En otro lugar he intentado mostrar cómo, en principio, es posible una concepción neutral de la misma ${ }^{33}$. De hecho, en un conocido texto de una carta del 25 de Enero de 1903 a W. E. Hocking Husserl escribe:

«La expresión 'los objetos se constituyen' en un acto significa siempre la propiedad del acto de representarse el objeto, jno 'constituirlo' en sentido propio! $\aleph^{34}$.

El mismo Husserl, después de las radicales afirmaciones derivadas del experimento mental de la aniquilación del mundo sostiene que todo aquel que objete que dichas afirmaciones llevan, en última instancia, a echarse en los brazos de un idealismo berkeleyano no ha entendido el sentido de lo que quería decir. No se defiende pues que el esse de la realidad sea su percipi,

«[...] sino que se desecha una interpretación de ella que entraña un contrasentido o que contradice su propio sentido aclarado con evidencia intelectual. Esta interpretación nace de un hacer filosíficamente absoluto el mundo, que es algo completamente extraño a la manera natural de considerar el mundo. Esta manera es, justamente, natural, vive ingenuamente en el llevar a cabo de la tesis general descrita por nosotros, no puede resultar nunca, pues, un contrasentido. Éste sólo brota cuando se filosofa, y buscando una última revelación sobre el sentido del mundo, no se nota que el mundo mismo tiene todo su ser como un cierto 'sentido' que presupone la conciencia absoluta como campo del dar sentido $[\ldots]\rangle^{35}$.

32 Ibid.

33 Cf. M. Crespo, «¿Hay una teoría no idealista de la constitución?», Revista de Filosofía, Vol. 65 (2009), 105-114.

34 «Der... Ausdruck, daß sich in einem Akte 'Gegenstände konstituieren', besagt immer die Eigenschaft des Aktes, den Gegenstand vorstellig zu machen: nicht 'konstituieren' im eigentlichen Sinn!» (Citado por U. PANZER en E. HusserL, Logische Untersuchungen. Zweiter Teil. Untersuchungen zur Phänomenologie und Theorie der Erkenntnis. In zwei Bänden. Halle 1901; rev. ed. 1922. The Hague, Netherlands: Martinus Nijhoff, 1984, p. XVIII, nota 2.

35 E. Husserl, Ideen qu einer reinen Phänomenologie, Erstes Buch, Hua III/1, \55. 
En palabras de Stein,

«Sin duda es absurdo hablar de un ser que no puede ser experimentado en principio. Pero es absurdo, no porque 'ser' no implique sino ser experimentado o al menos poder ser experimentado, sino porque lo que no es espiritual no puede ser a partir de sí mismo [...], sino que sólo puede ser como creado. Lo personal-espiritual, sin embargo, por ser, es consciente de sí mismo y, por crear lo que es distinto de sí mismo, sabe de aquello de lo que es distinto» ${ }^{36}$.

De este modo, como sostiene Robert Sokolowski, la relatividad de la realidad a la subjetividad significa que aquella depende de ésta para su sentido, que «la subjetividad es lo que da sentido, es la fuente de sentido y dado que la realidad tiene un sentido, ésta es dependiente de y relativa a la conciencia» ${ }^{37}$. En resumen, las cosas reales, en cuanto son unidades de sentido, presuponen la subjetividad como su correlato, ya que el sentido sólo puede surgir en conexión con la subjetividad intencional. Esto significa que

«si la realidad no puede ser concebida aparte de la correlación con la subjetividad, esto implica al menos que la conciencia es una condición sine qua non para lo real. La conciencia es un fundamento para el mundo en el sentido de que el mundo no puede llegar a ser 'real' (verum) a no ser que haya una conciencia ${ }^{38}$.

Contra esta afirmación no hay nada que objetar ni desde la perspectiva steiniana, en particular, ni, a mi juicio, desde una perspectiva realista, en genera ${ }^{39}$. Dicho en términos positivos -y éste es, a mi entender, uno de

36 E. STEIN, Excurso sobre el idealismo transcendental, 39.

37 R. Sokolowski, The Formation of Husserl's Concept of Constitution, Martinus Nijhoff, The Hague, 1970, 129.

38 R. Sokolowski, The Formation of Husserl's Concept of Constitution, 137.

39 Peter Volek distingue dos formas de idealismo trascendental. Por un lado, tendríamos una forma «moderada» (gemässigte Form), según la cual las cosas son constituidas cognoscitivamente (erkenntnismässig) por la subjetividad y, por otra, una forma «en sentido propio» (eigentliche Form). De acuerdo con esta última, el mundo es constituido ontológicamente (seinsmässig) por la subjetividad. Comparto con Volek que el idealismo trascendental en sentido estricto sostiene la constitución ontológica del mundo, pero tengo serías reservas acerca de que lo que el denomina «forma moderada» de idealismo trascendental sea realmente una forma de idealismo. Cf. P. VoleK, Erkenntnistheorie bei Edith Stein. Metaphysische Grundlagen der Erkenntnis bei Edith stein im Vergleich zu Husserl und Thomas von Aquin, Europäische Hochschulschriften, Bd. 564, Peter Lang, Frankfurt am Main 1998, 61. 
los aportes metodológicos más significativos de Edith Stein- el estudio del modo en el cual surgen los objetos ante la conciencia no significa necesariamente sostener un idealismo trascendental.

No es éste, pues, el punto en el que Stein y sus colegas de Gotinga vieron el inicio del camino de Husserl al idealismo trascendenta $l^{40}$, sino, más bien, en una consecuencia extraída de él. Stein se refiere a ello en un significativo texto:

[Lo que] «extrañó en el propio círculo de amigos y discípulos de Husserl era una consecuencia - a nuestro modo de ver innecesaria- que él sacó del hecho de la constitución: si determinados procesos regulados de la conciencia llevan a que al sujeto se le presente un mundo objetual, entonces el ser objetual significa [...] nada más que el ser dado para una conciencia de tal y tal manera $[\ldots]\rangle^{41}$.

El paso, pues, al idealismo trascendental en sentido propio consistiría en la consideración de que ser-real no significa otra cosa que ser-experimentado, que el ser del mundo no es sino su aparecer. En un texto de madurez Stein escribe:

«Pero no es absurdo decir que el ser del mundo no es lo mismo que tal aparecer, ni que es concebible otra manera de conocer el mundo y la existencia del mundo material ante Dios antes de que hubiese criaturas vivientes bajo cuyos sentidos el mundo pudiese caer. Y ser creado

40 Sokolowski insiste justamente en este punto: «Within the fundamental phenomenological meditation in Ideas, we already have some reason to choose the first alternative, and to say that consciousness is only a necessary condition for reality to appear. Our choice is based on the profound distinction Husserl makes between consciousness and reality; between the two there stretches an 'abyss' of sense. They are fundamentally, irreducibly different. They have a different ontic status. But if we were to say that subjectivity causes the sense and meaning found in reality, then his radical distinction would vanish. The senses and meanings in reality would have the same generic status as subjectivity has, because they would simply be effects or emanations from consciousness. In other words, the enigma of transcendence would be destroyed. The transcendence and objectivity of real sense would be dissolved into a projection of subjectivity. To avoid this, to maintain the proper nature of transcendent subjectivity, we must choose the first possibility. If subjectivity is only a condition for the emergence of sense, then the objective transcendence of sense can be maintained» (The Formation of Husserl's Concept of Constitution, p. 138).

41 E. Stein, Welt und Person: Beitrag zum christlichen Wabrheitsstreben. Edith Stein Werke, VI, Louvain: Nauwelaerts 1962,10ss. 
significa ser puesto fuera de Dios y tener un ser distinto del ser en la mente divina» ${ }^{42}$.

Es, por tanto, una determinada interpretación del darse de los objetos ante la conciencia la que apartó a Edith Stein y a sus colegas de la orientación del maestro. Ella consideró que esta interpretación era más bien una «convicción metafísica personal»y no «el resultado de una investigación fenomenológica». En este orden de cosas, uno de los méritos metodológicos de Stein es haber pensado sobre el modo en el que las cosas se nos dan y en nuestra habilidad de dejar que éstas se nos den. De este modo, no sólo pensamos sobre las cosas que nos son dadas en la experiencia, sino también en nosotros mismos como pensándolas. Vistas así las cosas, la fenomenología no sería sino - en palabras de Sokolowski- «el autodescubrimiento de la razón en la presencia de los objetos inteligibles» ${ }^{43}$.

Relacionado con esto, Edith Stein es especialmente consciente de que los distintos tipos de objetos tienen distintos modos de darse. No son iguales, por ejemplo, los aspectos de un objeto temporal, de una palabra, de una frase, de un suceso que los aspectos de un objeto especial como, por ejemplo, un cubo. Cada tipo de cosa prescribe sus series particulares de apariencias (fenómenos) en las cuales puede ser identificada. Por consiguiente, la cosa gobierna sus propias apariencias en las cuales es reconocible como lo que es. Así, la distinción entre lo psíquico y lo físico, por ejemplo, no es sólo una distinción del modo de darse, sino más bien de «una separación de objetos de diverso modo de ser a los que corresponde, por legítima esencia, un modo diferente del darse ${ }^{44}$. No es pensable, pues, teoría alguna de la conciencia que no incluya una teoría de los objetos que, en sus múltiples formas, son entendidos por la conciencia.

Volviendo brevemente a la cuestión del idealismo trascendental de Husserl y sin ánimo de entrar en una discusión detallada de este punto, resulta sumamente interesante considerar las reflexiones del filósofo español Antonio Millán-Puelles en su obra La estructura de la subjetividad. En ella se defiende que lo característico del idealismo trascendental no

42 E. STEIN, «Exkurs über den transzendentalen Idealismus» en E. STEIN, Potenz und Akt, Edith Steins Werke, Band XVIII, Verlag Herder GmbH, Freiburg, Basel, Wien 1998, 246-258 (Excurso sobre el idealismo trascendental, trad. W. Redmond, Opuscula philosophica, 20, 2005, 39).

43 R. Sokolowski, Introduction to Phenomenology, Cambridge University Press, Cambridge 2000, 4.

44 E. STEIn, Sobre el problema de la empatía, Trotta, Madrid 2004, 45. 
sería tanto el haber sostenido la existencia de una conciencia absoluta, sino el no haber distinguido entre conciencia y subjetividad y el pensar esta última como un simple «momento estructural» de la primera ${ }^{45}$. Dicho en palabras de este autor, «el ego que está implícito en el cogito queda así reducido a la condición de un mero 'polo funcional' del pensamiento»" De este modo, la subjetividad queda reducida a su conciencia. Ahora bien, afirma Millán-Puelles, «si se admite para la interna posibilidad de una conciencia la noción de una subjetividad distinta de ella, el idealismo queda destruido en su propia raíz» ${ }^{47}$. El idealismo husserliano sería más bien un idealismo fenomenológico, pero no un absoluto idealismo.

No puedo entrar aquí en esta interesante cuestión, entre otras cosas porque aún he de pensar más sobre ella. En cualquier caso, déjenme decirles que parece que la mayoría de los fenomenólogos realistas han emprendido más bien el camino de la metafísica del objeto dejando de lado el camino de la metafísica del sujeto. Salvo quizá la segunda parte de su Introducción a la filosofía la gran parte de los análisis fenomenológicos de Stein se refieren a objetos.

Con independencia de las diversas interpretaciones de su desarrollo histórico, creo que una de las virtualidades de la fenomenología -no exclusiva, ciertamente, de ella- es haber puesto de manifiesto este punto, a saber, el que las cosas se dan de una determinada forma porque son de una determinada forma. De hecho, en la lección inaugural de Husserl en la Universidad de Friburgo y frente al «paisaje filosófico» de esa época, éste plantea la necesidad de una «filosofía completamente originaria». Esta originariedad pasa por un tomarse en serio las apariencias o, si se prefiere, los fenómenos. En palabras de Robert Sokolowski,

«la fenomenología insiste en que la identidad e inteligibilidad están en las cosas y que nosotros somos aquellos ante los cuales tales identidades e inteligibilidades son dadas. Podemos evidenciar el modo en el que las cosas son. Cuando hacemos esto, descubrimos objetos, pero también nos descubrimos a nosotros mismos, precisamente como datives of disclosure, como aquellos a los cuales las cosas aparecen ${ }^{48}$.

\footnotetext{
45 A. Millán-Puelles, La estructura de la subjetividad, Ediciones Rialp, Madrid 1967, 129.

46 Ibid.

47 A. Millán-Puelles, La estructura de la subjetividad, 130.

48 R. Sokolowski, Introduction to Phenomenology, 4.
} 
Relacionado con el tema de nuestro simposio, cabría preguntarse hasta qué punto la condición femenina influye en este reconocimiento del sujeto como «dative of disclosure», como instancia ante la cual las cosas aparecen. Si existiera algún tipo de especificidad al respecto, ¿se trataría de un elemento eidético o de una simple cuestión fáctica, que es así, pero podría ser de otro modo?

IV

Un último aspecto metodológico fundamental de Edith Stein al que quisiera referirme tiene que ver con su tratamiento de la persona. Mientras que la individualidad de la cosa material significa que es numéricamente una, en el caso de la persona también, pero va mucho más allá. La individualidad de ésta es una unicidad cualitativa, espiritual, no expresable en palabras, sino que es algo último, irreductible a las estructuras generales estudiadas tanto por la Psicología, la Filosofía como por las «ciencias del espíritu». En alguna medida, la Historia, la Filología o la Poesía expresan un cierto grado de individualidad, pero, a pesar de ello, sólo se refieren a ciertos sectores de lo real. De este modo, la persona queda como algo inexplicable en su individualidad cualitativa por ciencia alguna. A pesar de esta inefalibilidad de la individualidad personal, encontramos en el capítulo VIII de Ser finito y ser eterno algunas claves para comprender mejor en qué consiste ésta ${ }^{49}$. Así una consideración apresurada podría llevar a decir que con las personas sucede algo semejante a lo que pasa con las cosas materiales, las plantas, los animales, etc. Podríamos decir que el ser persona es la esencia común a todas las personas individuales. Ahora bien, resulta fácil ver que el ser individual de las personas (Einzelsein, en la terminología de Stein) es diferente del ser individual de las cosas no-persona${ }^{l e s}{ }^{50}$. Estas diferencias pueden ser percibidas en, al menos, dos respectos. Al primero de ellos se refirió expresamente Edith Stein mientras que para referirme al segundo utilizaré algunos elementos del análisis que de la individualidad personal ha realizado recientemente el filósofo norteamericano John Crosby.

49 Cf. E. SteIn, Endliches Sein und ewiges Sein. Versuch eines Aufstiegs zum Sinn des Seins, Edith Steins Werke. Band II, Hsg. V. L. Gelber und R.Leuven, Herder, Freiburg 1950, 431ss.

50 «Das allgemeine Wesen ist das, was das Einzelding mit keinem anderen teilt. Worin aber besteht das, was das Einzelding mit keinem anderen teilen kann und was es zum Einzelding macht?» (E. STEIN, op.cit., 431) 
Al primer respecto desde el cual analizar la individualidad de las personas humanas se refiere la propia Edith Stein cuando pone de manifiesto el hecho de que las personas humanas pueden decir yo a diferencia por ejemplo de esta mesa o del animal más evolucionado. Este decir yo entraña el percibirnos cada uno de nosotros como un individuo diferente de los demás y como capaz de construirnos a nosotros mismos ${ }^{51}$. Así, en la medida en que yo, por medio de mi libre actividad, me decido por esta o aquella posibilidad de actuación, me conformo a mí mismo, me construyo como la persona individual que soy. Esta individualidad no puede ser expresada por medio de términos generales. Es, como Stein afirma, unaussprechlich. Se trata, por así decir, de nuestro centro espiritual, de lo «profundo de nuestra alma» (Tiefe der Seele), el cual «impregna» cada una de nuestras características y cada una de nuestras acciones y que, observado en otras personas, es lo que en el fondo nos atrae o repele de ellas ${ }^{52}$.

El segundo respecto desde el cual abordar la individualidad personal y que, en este contexto, resulta a mi juicio relevante ha sido puesto de relieve por John Crosby. Este fenomenólogo norteamericano ha mostrado cómo la individualidad de las personas constituye, junto con la nota común a todas ellas de la racionalidad, una fuente de la dignidad humana ${ }^{53}$.

51 «Dass das Einzelsein des Menschen -wie jeder geistigen Person- sich vom Einselsein aller unpersönlichen Dinge unterscheidet, ist schon deutlich geworden: es gehört dazu, dass das Leben dem Ich entquillt und dass es dem persönlichen Ich auf doppelte Weise in die Hand gegeben ist: um sich seiner als eines von allem gesondertetn Lebens bewusst zu werden und um es frei zu gestalten» (E. STEIN, op.cit., 458).

52 «Und das Innerste der Seele, ihr Eigenstes und Geistigstes, ist kein farb- und gestaltloses, sonern ein eigentümlich geartetes: sie spürt es, wenn sie ,bei sich selbst', , in sich gesammelt ist. Es lässt sich nicht so fassen, dass man es mit einem allgemeinen Namen nennen könnte, es ist auch nicht mit andern vergleichbar. Es lässt sich nicht in Eigenschaften, Charakterzüge u. Dgl. Zerlegen, weil es tiefer liegt als sie: es ist das Wie (poion) des Wesens selbst, das seinerseits jedem Charakterzeug und jedem Verhalten des Menschen seinen Stempel aufprägt und dem Schlüssel zum Aufbau seines Charakters bildet. Durch diese ,Äusserungen“ wird das Innerste der Seele auch von aussen fassbar. Wir ,spüren'das Unasusprechliche ihres Wesens auch an andern. Es ist das, was uns im tiefsten Grund, anzieht' oder ,abstösst'. Wir können uns dabei wie von etwas Verwandten berührt fühlen. Aber meine Art und die des andern lassen sich nicht in etwas Gemeinsames und etwas Unterscheidendes auseinanderlegen. In diesem Sinn müssen wie zugestehen, dass der Wesensunterschied des Einzelnen nicht fassbar ist» (E. STEIN, op.cit., 458-459).

53 Cf. las siguientes obras de J.F. Crosby: «A Neglected Source of the Dignity of Persons». En J. F. Crosby, Personalist Papers, The Catholic University of America Press, Washington 2004, 3-32; The Selfhood of the Human Person, The Catholic University of 
Con ello no se quiere despreciar la riqueza y profundidad de la idea de racionalidad y su contribución a la fundación de la dignidad humana. Lo problemático de esta respuesta, según nuestro autor, es considerar que la naturaleza común a todos los seres humanos constituye la única fuente de dignidad. Los seres humanos no somos simplemente un ejemplar de una especie con características comunes a todos los otros ejemplares. Es más, la propia experiencia moral nos dice que nos sentiríamos «mal tratados» si únicamente se nos considerara como meras instancias del género humano o como meros portadores de propiedades o cualidades universales. Si, adaptando un ejemplo del propio Crosby, pudiéramos preguntar a los que conocieron y amaron a Edith Stein si todo lo que conocieron y amaron en ella puede ser repetido en otro ser humano, éstos nos responderían con un no. Insistirán en que en Edith Stein hubo algo absolutamente irrepetible, que ella no era un mero espécimen o instancia de modo que una Edith Stein es estricta, absolutamente, imposible. La Edith Stein incomunicable fue algo absolutamente inefable, algo demasiado concreto para los conceptos generales del lenguaje humano. Todos los que la conocieron en todo lo que le hacía irrepetible encontraron de hecho dignidad, valor en ella. De otro modo, no la hubieran amado como lo hicieron. Si en nuestro experimento mental Edith Stein, volviera a la vida, afirmaría lo siguiente: No es sólo porque comparto la naturaleza racional común a todos nosotros por lo que tengo dignidad, sino también porque soy la persona irrepetible que soy. Cualquiera que se familiarice conmigo en mi mismidad irrepetible se familiariza con una dimensión de mi dignidad que de otro modo se le escaparía ${ }^{54}$.

Ciertamente, este no es el lugar para profundizar en estas cuestiones tan relevantes. Tan sólo quisiera apuntar que, desde un punto de vista metodológico, el camino hacia la pregunta por la mujer debería, a mi juicio, hacer una «parada» en la cuestión acerca de la individualidad. ¿Hasta qué punto la condición femenina influye en la individualidad de las personas humanas de ese género o, por el contrario, se trata de una condición que está en un nivel metafísico diferente? ¿En qué sentido puede la condición femenina influir en mi mismidad irrepetible? El ser-mujer ¿influye en la construcción del sí-mismo por parte del yo? ¿Hasta qué punto podría hablarse de un «sí-mismo» femenino reconocible por medio de o a través de las acciones libres?

American Press. Washington 1996 (La interioridad de la persona bumana. Trad. de A. Herrera, Ediciones Encuentro, Madrid 2007).

54 J. Crosby, «A Neglected Source of the Dignity of Persons», ed. cit., pág. 12. 
La radicalidad del método filosófico de Edith Stein constituye un modelo innegable de reflexión filosófica acerca de las cosas mismas porque, a fin de cuentas, de lo que depende que nuestra aspiración a llamarnos filósofos se cumpla es de pensar sobre las cosas mismas y no de mostrar lo mucho o poco que sabemos sobre las teorías acerca de ellas. 
Resumen: este trabajo aborda tres aspectos fundamentales del método filosófico de Edith Stein. En primer lugar, se alude a las cosas mismas como el punto de partida del filosofar de esta autora. En segundo lugar, se considera el aspecto que constituye uno de los aportes fundamentales del método fenomenológico y que es claramente reconocible en nuestra autora, a saber, el haber puesto de manifiesto la imposibilidad de hacer filosofía primera sin tomar en cuenta la vida consciente ante la que todas las cosas se abren. En tercer lugar, se remite a la individualidad de la persona como un aspecto de la antropología de Stein especialmente relevante. Al final del análisis de cada uno de estos tres aspectos metodológicos se intenta mostrar en qué sentido éstos pueden ser de relevancia para emprender el camino hacia la pregunta por la mujer.

Palabras clave: Edith Stein, fenomenología, conciencia, individualidad, persona, mujer.

Abstract. This paper addresses three fundamental aspects of Edith Stein's philosophical method. Firstly it looks at issues such as the author's starting point of philosophizing. Secondly it considers the aspect which constitutes one of the fundamental contributions of the phenomenological method and is clearly recognizable in our author, such as having highlighted that it is impossible to carry out first philosophy without taking into account conscious life, before which all things are opened. Thirdly it looks at the individuality of the person as an especially relevant aspect of Stein's anthropology. After analyzing each of these methodological aspects, this paper attempts to show how they may be relevant in laying out the path towards the question of the woman.

Key words: Edith Stein, phenomenology, conscience, individuality, person, woman. 